\title{
DROUGHT MONITORING IN SHANDONG PROVINCE BASED ON MODIFIED TEMPERATURE-VEGETATION-SOIL MOISTURE DRYNESS INDEX (TVMDI)
}

\author{
Hong Wan ${ }^{1,2}$, Zhendong Wang ${ }^{1,2}$, Peng Guo ${ }^{1, *}$, Bo Wang ${ }^{1}$, Xican $\operatorname{Li}^{1}$, Chunhong Meng ${ }^{1}$, Gang Yang ${ }^{1}$ \\ 1. College of Information Science and Engineering, Shandong Agricultural University. Taian 271000, China; guopeng@sdau.edu.cn \\ 2. Institute of Remote Sensing and Digital Earth, Chinese Academy of Sciences, Beijing 100094, China;
}

KEY WORDS: Drought Monitoring, TVMDI, MODIS, FY-3B, Shandong

\begin{abstract}
:
Drought is one of the frequent natural disasters in Shandong province, which is characterized by high frequency and wide range. In response to frequent droughts that are not monitored in time, monitoring the changes of drought is of great significance to agricultural production and social development. This study used the Temperature-Vegetation-soil Moisture Dryness Index (TVMDI) model, combined with the optical MODIS land surface temperature, vegetation index, surface albedo data and microwave FY-3B soil moisture data, to monitor the drought of Shandong province in 2016. The precipitation and temperature data of weather station were used to validate the monitoring results. The results show that, in 2016, the drought in Shandong province mainly occurred in winter and spring, and the drought in summer was alleviated. From the perspective of space, the northern Shandong and the Shandong peninsula areas are relatively humid with less drought time, while the local areas in the central and southern Shandong province suffer from severe drought with longer drought time. From the perspective of correlation with meteorological factors, the average correlation coefficient between TVMDI and precipitation can reach 0.45 , and the average correlation coefficient between TVMDI and temperature can reach 0.63 .
\end{abstract}

\section{INTRODUCTION}

Drought is a frequent natural disaster in China. It not only damages the agricultural production and ecological environment, but also seriously affects the social production and life of human beings. In recent years, the occurrence of drought disasters in China has become increasingly frequent, so how to effectively and accurately monitor drought has become a research hotspot. Due to its advantages of low data acquisition cost, abundant data, short observation period and large scales, remote sensing has become the main means of drought monitoring. TVMDI is a new drought monitoring model proposed by Amani et al. [1]. The method can be used to monitor drought timely and effectively. Amani et al. pointed out that soil moisture (SM) was a direct indicator of drought, while land surface temperature (LST) and vegetation status were indirect indicators of drought. These three variables are related variables, and one of them change will cause change in the other two. In order to better understand the drought state, a TVMDI model which combined LST, PVI and SM on the threedimensional characteristic space of PVI, LST and reverse soil water formation. Based on the MODIS LST, vegetation index, surface albedo and the microwave FY-3B soil moisture data, this paper used TVMDI model to study the drought situation in Shandong province in 2016. Firstly Savitzky - Golay (S - G) filter method was used to reconstruct the MODIS data, then the MODIS data was used to downscale the FY-3B soil moisture. Finally, using optical remote sensing MODIS data and downscaled FY-3B soil moisture data, a three-dimensional feature space composed of normalized surface temperature, vegetation index and soil moisture, namely TVMDI, is constructed. The monthly average precipitation and temperature data of five national meteorological stations are used to analyse and validate the drought monitoring results. The results can provide a help for drought analysis, disaster prevention and mitigation in Shandong province.

\section{STUDY AREA AND DATA PREPROCESSING}

\subsection{STUDY AREA}

Shandong province is located in the eastern of China and the lower reaches of the Yellow River, with the longitude of $114^{\circ} 20$ ' 122 $43^{\prime} \mathrm{E}$, latitude of $34^{\circ} 23^{\prime} \sim 38^{\circ} 23^{\prime} \mathrm{N}$ and its area is $158,000 \mathrm{~km}^{2}$. Shandong province belongs to the temperate monsoon climate zone, summer is hot and rainy, winter is cold and dry, and the change of four seasons is obvious. The annual average temperature of the whole province basically follows the distribution rule of decreasing from southwest to northeast, but there is little regional difference. The average annual temperature is between $10 \sim 14^{\circ} \mathrm{C}$, and the average annual precipitation is $550 \sim 950 \mathrm{~mm}$. Fig. 1 shows the elevation map of Shandong province and the distribution of meteorological stations. It can be seen from the figure that the central and eastern part of Shandong province is mountainous and hilly, with relatively undulating terrain. The western and northern areas belong to the north China plain, flat terrain.

\footnotetext{
* Corresponding author
} 


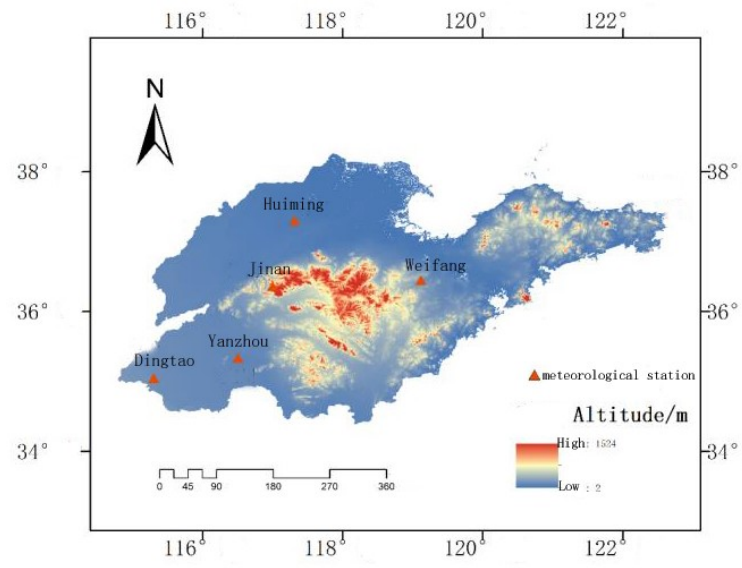

Figure 1 location of study area and meteorological stations

\subsection{DATA SOURCES AND PREPROCESSING}

\subsubsection{MODIS data and preprocessing}

In this study, four MODIS data, including surface albedo product MCD43B3, land surface temperature product MOD11A2, vegetation index product MOD13A2, and surface reflectivity product MOD09Q1, was used. The time range of data is from January to December 2016. All products are downloaded from the MODIS website (https://ladsweb.modaps.eosdis.nasa.gov/), these products undergo radiometric and geometric correction and are projected as sinusoidal projections in $\mathrm{HDF}$ format. MOD11A2 is an 8-day synthetic product with a spatial resolution of $1 \mathrm{~km}$, and MOD13A2 is a 16-day synthetic product, which includes NDVI and EVI vegetation index. MOD43B3 has a spatial resolution of $1 \mathrm{~km}$ and is synthesized in 16 days. MOD09Q1 consists of two bands, red band and near-red band, with a spatial resolution of 250 meters and a time interval of 8 days.

First, the MODIS data is reprojected, resampled and format conversion using the MRT (MODIS Reprojection Tools) provided by NASA. After pre-processing, 46 surface temperature images, 23 vegetation index images, 46 red band and near-red band reflectivity images and 46 surface albedo images of Shandong province in 2016 were obtained. According to formula 1 , the modified soil adjusted vegetation index (MSAVI) can be calculated using band red band and NIR data. The spatial resolution is $250 \mathrm{~m}$, and the image with a resolution of $1 \mathrm{~km}$ can be obtained through resampling.

$$
M S A V I=\frac{2 N I R+1-\sqrt{(2 N I R+1)^{2}-8(N I R-R E D)}}{2}
$$

\subsection{2 $\quad$ FY-3B soil moisture products}

FY-3B data were downloaded from the national satellite meteorological center, and there are two kinds of soil moisture products, namely ascending orbit (day) and descending orbit (night), covering the whole world. The data adopts the equal area scalable earth grid projection, the data format is HDF5, and the spatial resolution is $25 \mathrm{~km}$.

\subsubsection{Meteorological data}

The precipitation data and temperature data used in this paper are from National Meteorological Information Center (http://data.cma.cn/). In this study, monthly average precipitation and temperature data of five national meteorological stations including hui-min in, dingtao, jinan, yanzhou, and weifang were selected ${ }^{[3]}$. The data time range is from January to December 2016, and the location information of the weather station is shown in figure 1 .

\subsection{Data reconstruction}

The remote sensing data is interfered by various factors such as sensor deviation, atmospheric radiation, electromagnetic interference, etc. As a result, there will be a lot of noise in the image, which reduces the image quality. The S-G time sequence filtering and spatial filtering methods proposed by Savitzky and Golay $^{[4]}$ can be well applied to the reconstruction of NDVIIEVI and other data, and can smooth the vegetation index curve well, fully reflecting the vegetation changes. The weighting coefficient in S-G filter depends on the least squares fitting times of a given higher-order polynomial in a filter window. If a data set contains $n$ points, the width of half window is $m$. The calculation formula of S-G filtering algorithm is as follows:

$$
Y_{j}^{*}=\frac{\sum_{i=-n}^{i=m} C_{i} * Y_{j+1}}{N}
$$

Where, $\mathrm{Y}_{\mathrm{j}}^{*}$ is the result after smoothing; $\mathrm{Y}$ is the original data; $\mathrm{C}_{\mathrm{i}}$ is the weight of each point in the smoothing window; $\mathrm{N}$ is the length of the filter, which is also the width of the sliding array; $\mathrm{j}$ represents the JTH point. In the calculation process, pixel reliability and weight selection Quality control files provided by MODIS data are used to assign different weights to each pixel in the original data that needs filtering.

\section{DROUGHT MONITORING BASED ON TVMDI}

\subsection{Soil Moisture downscaling}

As the resolution of FY-3B soil moisture product is only $25 \mathrm{~km}$, it cannot directly build model with the vegetation index, LST and other data with the resolution of $1 \mathrm{~km}$, so it is necessary to downscale soil moisture data to achieve the consistency of all the data. In this paper, FY-3B low-resolution soil moisture data were downscaled using multiple statistical regression method ${ }^{[5]}$. This method assumes that models at different scales have the same relationship, elevates the high-resolution MODIS data to a lower resolution, establishes a model with the low-resolution soil moisture, and obtains the model coefficient through regression analysis. Then, applying the model to the highresolution data, the high-resolution soil moisture can be obtained ${ }^{[6]}$.

Chauhan $^{[7]}$ et al. proposed a method to combine optical data, near-infrared data and passive microwave data. In order to reduce the dependence of LST, vegetation index, Albedo and other parameters on the external environment, and to facilitate the comparison between different regions, a linear model of soil moisture and normalized difference vegetation index (NDVI), LST and surface Albedo was established.

$$
\begin{aligned}
S_{m}= & a_{000}+a_{001} A^{*}+a_{010} T^{*}+a_{100} N D V I^{*}+a_{002} A^{* 2} \\
& +a_{020} T^{* 2}+a_{200} N D V I^{* 2}+a_{011} T^{*} A^{*} \\
& +a_{101} A^{*} N D V I^{*}+a_{110} N D V I^{*} T^{*}
\end{aligned}
$$

Where, $A^{*}$ represents normalized surface albedo, NDVI* represents normalized vegetation index, and $\mathrm{T}^{*}$ represents normalized surface temperature. The process of "normalization" is:

$$
T^{*}=\frac{T-T_{\min }}{T_{\max }-T_{\min }}
$$




$$
\begin{gathered}
N D V I^{*}=\frac{N D V I-N D V I_{\min }}{N D V I_{\max }-N D V I_{\text {min }}} \\
A^{*}=\frac{A-A_{\min }}{A_{\max }-A_{\min }}
\end{gathered}
$$

According to the multiple linear regression model, the downscaled soil moisture data in Shandong province in 2016 and the original FY-3B soil moisture data are shown in figure 2. The left image in Fig. 2 is the original FY-3B soil moisture, and the right is the downscaled result. Compared with the original data, the downscaled result basically maintained the original spatial characteristics of soil moisture, but the maximum value of soil moisture decreased, and the soil moisture in some areas was low. The reason for this phenomenon is that when satellites acquire soil moisture data, the data results are greatly affected by temperature changes. When downscaling, the average of temperature is selected to weaken the influence of temperature. Therefore, the data after down scaling is lower than the original data.

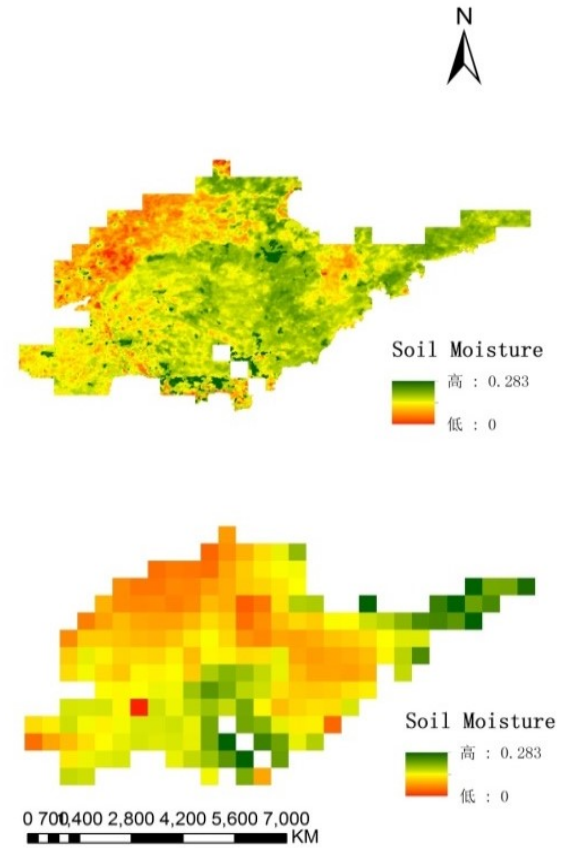

Fig.2 comparison of FY-3B /MWRI original and downscaled soil moisture

In order to further verify the feasibility of downscaling results, correlation analysis was conducted between monthly mean precipitation of Jinan meteorological station and FY-3B soil moisture before and after downscaling. As shown in figure 3, the correlation coefficient $\left(\mathrm{R}^{2}\right)$ between FY-3B original monthly soil moisture data and the measured precipitation is 0.83 , while the correlation coefficient $\left(\mathrm{R}^{2}\right)$ between the downscaling soil moisture results and the measured precipitation is 0.86. The results show that the soil moisture still maintains a good accuracy after downscaling.
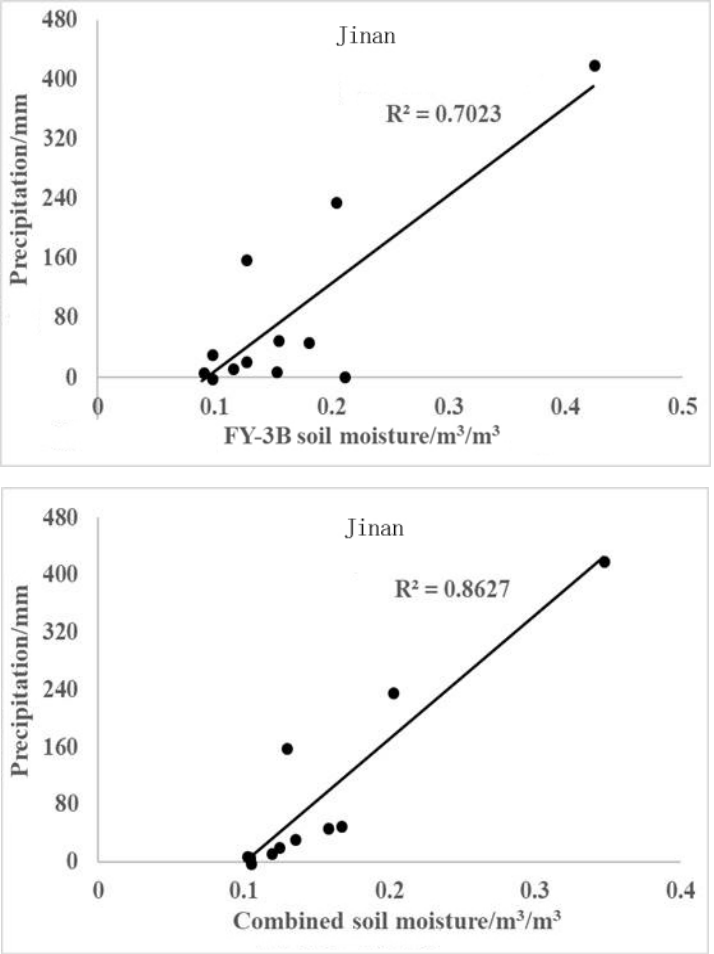

Fig. 3 correlation analysis between measured precipitation and original and downscaling soil moisture

\subsection{Drought monitor use TVMDI}

Soil moisture and vegetation condition are the most direct and important indicators of drought events. In real-time drought monitoring, the severity of drought can be predicted by the proximity of soil moisture and soil spectral reflection values. TVMDI is calculated from the normalized three-dimensional characteristic space composed of LST, vegetation index(VI) and soil moisture(SM), the calculation formula is as follows:

$$
T V M D I=\sqrt{L S T^{2}+S M^{2}+\left(\frac{\sqrt{3}}{3}-V I\right)^{2}}
$$

In previous studies, researchers only choose Perpendicular Vegetation Index(PVI) to calculate TVMDI, but PVI is sensitive to the variation of soil moisture. There are several other indices that are insensitive to variations of soil moisture, such as the Soil Adjusted Vegetation Index (SAVI) and the Modified Soil Adjusted Vegetation Index (MSAVI) ${ }^{[8]}$.By analyzing the correlation between PVI, MSAVI and NDVI, as shown in figure 4, it was found that MSAVI had a higher correlation. Therefore, MSAVI was selected in this study to calculate TVMDI.

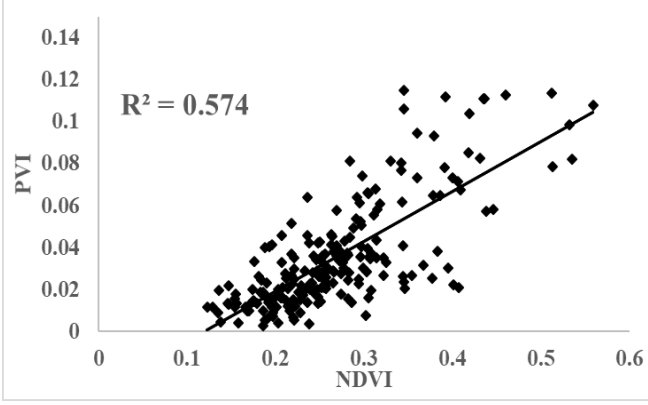




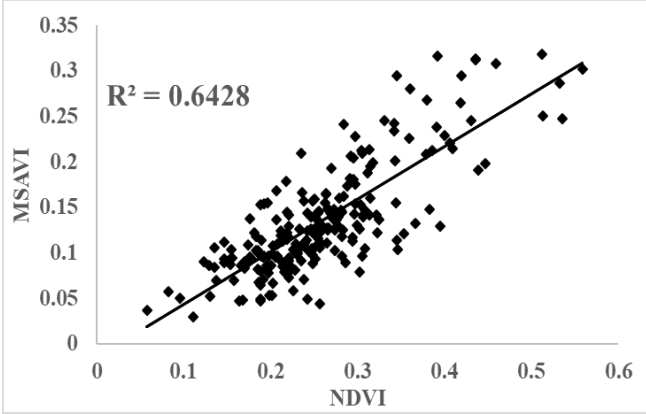

Fig.4 correlation comparison between PVI/MSAVI and NDVI

\subsection{Result analysis}

Soil moisture, land surface temperature and vegetation index are correlated with each other, and changes in one variable will lead to corresponding changes in the other two variables. Generally speaking, the increase of soil moisture and vegetation coverage will lead to the increase of vegetation index and the decrease of TVMDI. Similarly, when soil moisture decreases and land surface temperatures rise, crops are affected by drought because there is not enough moisture in the soil, and the TVMDI value will rise. With the increase of land surface temperature, decrease of vegetation index and decrease of soil moisture, drought will become more serious and TVMDI will gradually approach its maximum value of 1 .

As shown in figure 5, in terms of time, drought occurred in most time of the winter and spring in Shandong province, during this period soil moisture was less, vegetation index was lower, and drought index was higher. In summer and autumn, more precipitation causes the increase of soil moisture and vegetation index, which makes the drought index lower and the degree of drought lighter. From the perspective of space, since the beginning of winter, a large area of Shandong province has been in a state of drought. It can be seen from the figure that the mountain and hilly areas of Shandong province are in a serious drought. The drought degree of northwest Shandong, west Shandong and peninsula area is low.

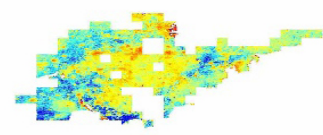

Jan.

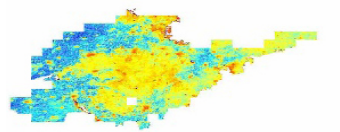

Mar.

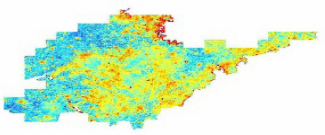

May.
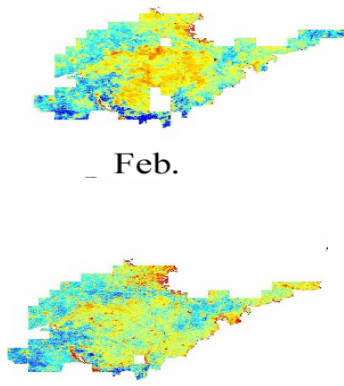

Apr.

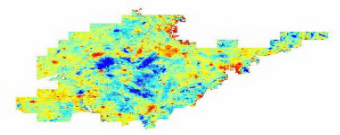

June.

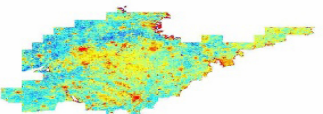

July.

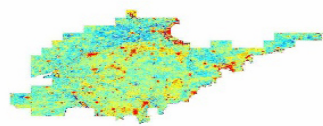

Sep.

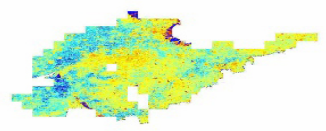

Nov.

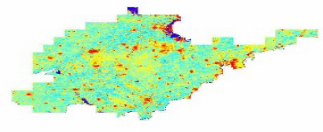

Aug.

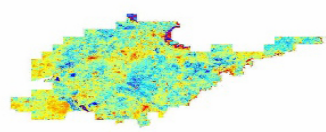

Oct.

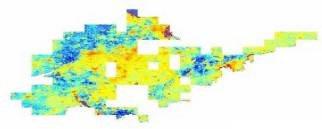

Dec.

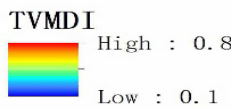

Fig.5 improved TVMDI figure of each month in Shandong province

Finally, correlation analysis was conducted between TVMDI and precipitation and temperature data of five surface meteorological stations shown in Fig. 1, the results were shown in table 1. According to table 1, the average correlation coefficient between TVMDI and precipitation is 0.45 , and the average correlation coefficient between TVMDI and temperature is 0.63 , and both of them have passed the significance test at the level of 0.01 . According to figure 5 , the temperature in winter is low, but the TVMDI value is high, while the temperature in summer is rising, and the TVMDI value is low. Generally speaking, drought is easy to occur when the temperature is high, and the verification results also show that TVMDI has a high correlation with the temperature. This may be caused by: on the one hand, the surface temperature is included in the calculation of TVMDI, which has a higher correlation with the temperature; on the other hand, the change of temperature in space is not as high as the change of precipitation. The data of meteorological stations selected in this paper are the average data in the buffer zone around meteorological stations, which only represents the real meteorological situation in a small area, and precipitation is higher, vegetation growth well with high vegetation coverage in summer. So even when summer temperatures are highest throughout the year, droughts are lower.

Tab.1 correlation coefficients between temperature, precipitation and TVMDI

\begin{tabular}{|c|c|c|}
\hline $\begin{array}{c}\text { meteorological } \\
\text { stations }\end{array}$ & $\begin{array}{c}\text { Correlation with } \\
\text { temperature }\end{array}$ & $\begin{array}{c}\text { Correlation with } \\
\text { precipitation }\end{array}$ \\
\hline Weifang & 0.62 & 0.44 \\
\hline Yanzhou & 0.44 & 0.40 \\
\hline Dingtao & 0.52 & 0.35 \\
\hline Huimin & 0.65 & 0.52 \\
\hline Jinan & 0.92 & 0.55 \\
\hline
\end{tabular}



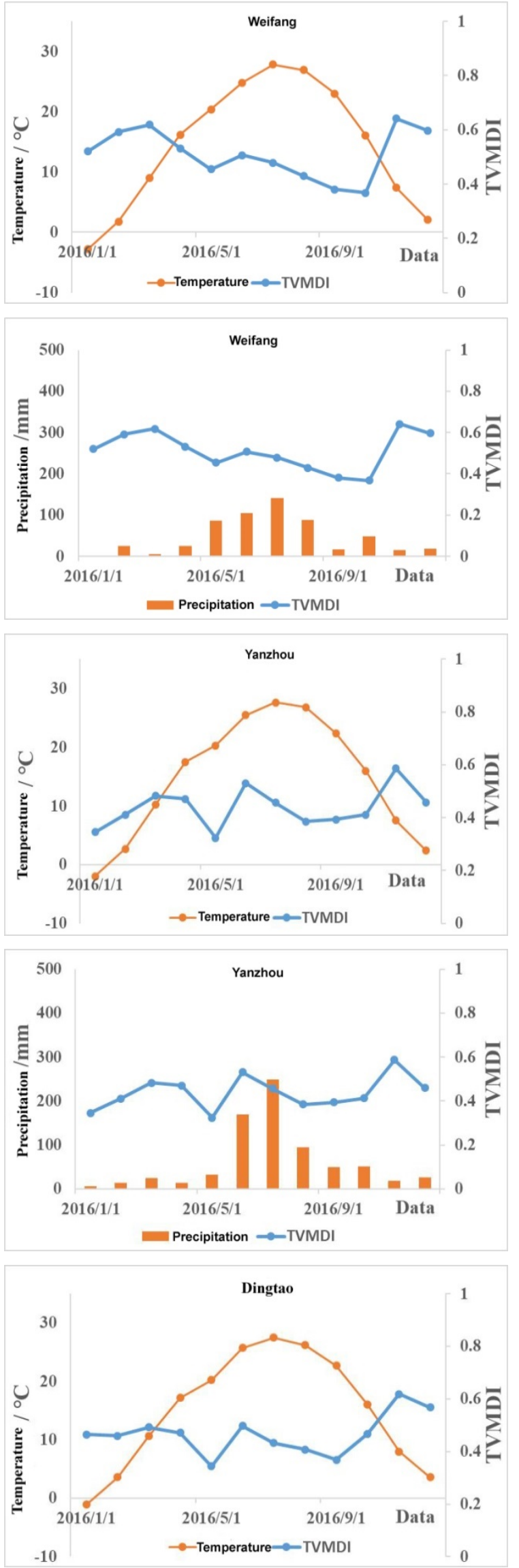
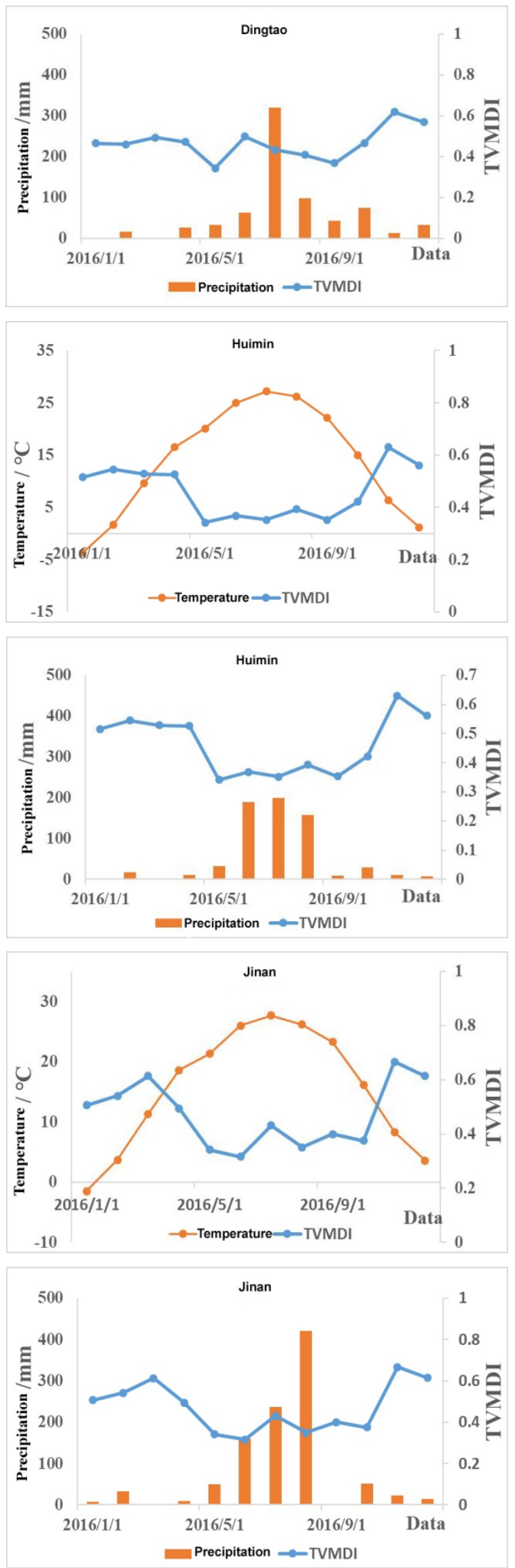

Fig.6 relationship between TVMDI and precipitation and temperature 


\section{CONCLUSION}

In this study newly improved TVMDI model based on the three-dimensional characteristic space of soil moisture, vegetation and surface temperature was used to monitor the drought situation in Shandong province in 2016.The conclusions are as follows:

(1) The MSAVI can effectively eliminate the influence of soil background and correct the shortcomings that some vegetation indexes cannot correctly reflect the real situation of the surface when the soil moisture is high.

(2) The average correlation coefficient between TVMDI and precipitation could reach 0.45 , and the average correlation coefficient between TVMDI and temperature could reach 0.63. This indicated that the improved TVMDI can be used to monitor drought in Shandong province.

(3) According to the results of the improved TVMDI model, the drought in Shandong province mainly happened in spring and winter, especially in February and March. In summer, due to the increase in precipitation, most parts of the province are relatively wet. In space, the mountainous areas and hills in Shandong are relatively dry, while the surrounding plain is relatively wet.

\section{ACKNOWLEDGEMENTS}

This study is jointly sponsored by Shandong Natural Science Foundation (Grant NO.ZR2015DL003) and the National Natural Science Foundation of China (Grant NO.41501409).

\section{REFERENCES}

[1]Amani M , Salehi B , Mahdavi S , et al., 2017: TemperatureVegetation-soil Moisture Dryness Index (TVMDI)[J]. Remote Sensing of Environment, 197:1-14.

[2] Qi J, Chehbouni Al, Huete A, Kerr Y, 1994: A modified soil adjusted vegetation index (MSAVI). Remote Sensing Of Environment, 48, 119- 126.

[3] WANG Zhengdong, GUO Peng, WAN Hong, YANG Gang. 2019: Analysis of Drought Monitoring in Shandong Province from 2014 to 2016 Based on MODIS Data (in Chinese)[J].Research of Soil and Water Conservation, 26(02): 330-336.

[4] A. Savitzky and M. J. E. Golay, "Soothing and differentiation of data by simplified least squares procedures," Anal. Chem.vol. 36, pp. 1627-1639, 1964.

[5] ZHOU Zhuang, ZHAO Shaojie, JIANG Lingmei.2016: Downscaling methods of passive microwave remote sensing of soil moisture[J]. Journal of Beijing Normal University(Natural Science),52(04): 479-485.

[6] Tuttle S E, Salvucci G D.2014: A new approach for validating satellite estimates of soil moisture using large-scale precipitation: Comparing AMSR-E products [J]. Remote Sensing of Environment, 142(4): 207-222.

[7] Chauhan, N.S., S. Miller, and P.2003: Ardanuy, Spaceborne soil moisture estimation at high resolution: a microwave-
optical/IR synergistic approach. International Journal of Remote Sensing, 24(22): p. 4599-4622.

[8] Huete A R . 1988: A Soil-Adjusted Vegetation Index[J]. Remote Sensing of Environment, 27(3). 